\title{
Evaluation of red meat allergy patients and review of the literature
}

\author{
Şeyhan Kutluğ $\breve{ }^{\oplus}$, Gonca Hancıoğlu${ }^{\oplus}$, Şefika İlknur Karadağ Kökçü®, Recep Sancak \\ Fadıl Öztürk ${ }^{\odot}$
}

Division of Pediatric Allergy and Immunology, Ondokuz Mayls University Faculty of Medicine, Samsun, Turkey.

\begin{abstract}
Background. It was reported that prevalence of red meat allergy in children was higher in our country than in western populations. However, the diagnosis of these patients is often delayed. The aim of the study was to present the clinical and laboratory characteristics of our red meat allergy patients.
\end{abstract}

Methods. The data were collected retrospectively from the files of children with red meat allergy. Also, 6 adults with red meat allergies were recorded in the families of the children. Patients with symptoms associated with red meat allergy and sensitive to beef or mutton in prick-to-prick tests were recorded.

Results. The median age of the 43 patients was 12 years (2-37), and 51\% were male. Most of the patients were children ( $n=37,86 \%)$. The median age was 10 years in children (2-17), and 54\% were male. All of the children had dermatologic manifestations, $51 \%$ had respiratory symptoms, and $64 \%$ had anaphylaxis upon exposure to red meat. The anaphylaxis history was not associated with demographic, clinical and laboratory data. A total of $63 \%$ children had additional allergic diseases, and $75 \%$ of them were sensitive to both mutton and beef in prickto-prick tests. The median total IgE level of the children was 327 (20-3550) IU/mL, median eosinophil count was $210 / \mathrm{mm}^{3}$ (40-990) and mean vitamin D was $13.1 \pm 1.2 \mathrm{mcg} / \mathrm{L}(\mathrm{n}=27)$. Anaphylaxis occurred in 3 of 9 patients who received the open oral food challenge (OFC) test. After OFC, 3 patients continued to eat red meat without issues, and 1 patient was recommended to eat alternatives to red meat.

Conclusions. Clinical and laboratory findings were heterogeneous in children with red meat allergy. Anaphylaxis risk seems to be higher than other food allergies. OFC test is more helpful in both diagnosis and alternative red meat selection compared to laboratory findings.

Key words: anaphylaxis, children, food allergy, oral food challenge, red meat allergy.

Food allergy is an important disease because it is associated with high morbidity in children and adults. ${ }^{1}$ It can cause nutritional deficiency and related consequences in children. ${ }^{2,3}$ Also, the quality of life in all food-allergic patients and their families is negatively affected. ${ }^{2}$ The frequency of IgE-mediated food allergy in children varies among countries and age groups, but is reported to be between 3.5\% and $11 \%{ }^{2}$ Food allergies have increased both in the

\footnotetext{
Şeyhan Kutluğ

seyhankutlug@hotmail.com
}

Received 21st July 2020, revised 9th December 2020,

11st January 2021, accepted 20th February 2021. general population and in children over the years, ${ }^{2,4}$ and is becoming increasingly important because they constitute the most common cause of anaphylaxis in children. ${ }^{5}$

In Western societies, peanut, egg, sesame and milk have been found to be most responsible for food allergies in children. ${ }^{2}$ Red meat allergy is rarely reported in children with food allergy. ${ }^{6,7}$ However, this is not the case all over the world. Foods responsible for allergies vary according to eating habits, geographic regions and race..$^{2,7,8}$ There is no prevalence study on food allergy in children covering the entire country of Turkey. In some studies conducted in Turkey regarding the prevalence of food allergy in children, red 
meat allergy was rarely reported, ${ }^{9-11}$ while in others it was frequently reported. ${ }^{12,13}$ The conflicting results may be due to the fact that these studies were conducted in different regions of Turkey. Red meat allergy is reported more frequently in the Black Sea region of Turkey. ${ }^{12,14-16}$ In a single-center study on the prevalence of red meat allergy in children in the black sea region, the primary beef allergy prevalence was reported to be $0.3 \%$, based on the oral food challenge (OFC) test. ${ }^{16}$ In a multicenter study in Turkey, it has been reported that beef is the second food responsible for anaphylaxis in children after milk. ${ }^{15}$ For this reason, it is critical to identify patients with red meat allergy to prevent potentially lifethreatening reactions at least in regions where red meat allergies are common. Patients with red meat allergy are diagnosed late, and even patients diagnose themselves in population where red meat allergy is rare. ${ }^{17}$ It is also argued that red meat allergy is responsible for some spontaneous and idiopathic anaphylaxis. ${ }^{18}$ For this reason, we want to present the features of our red meat allergy patients and raise awareness of red meat allergy by pediatricians. The aim of the study was to present the characteristics of our red meat allergy patients and review of the literature.

\section{Material and Methods}

\section{Study Population}

This retrospective study was conducted between January 2014 and December 2017 based on the records of patients admitted to Pediatric Allergy and Immunology Department. From these records, the individual that had been filled with any complaints after red meat consumption were selected. The records of the children whose complaints were associated with eating red meat and whose red meat sensitivity were shown with skin-prick tests were included in this study. Also, as we learned from the patients' clinical history, the files of patients over the age of 18 years in the same family with similar symptoms were included. Those who had taken other foods together with red meat, and who had described oral allergy syndromes were not included in the study. In the clinical history, it was called early-anaphylaxis if anaphylaxis developed at 0-2 hours after food intake, and delayed-anaphylaxis if anaphylaxis developed at 4-6 hours. ${ }^{19}$ The study was approved by the Ondokuz Mayis University Ethics Committee of our institution (KAEK 2017/55). The demographic data of the patients, clinical history and characteristics, laboratory findings and any data that led to diagnosis were recorded from the files.

\section{Laboratory Tests}

\section{Skin Prick Tests}

Firstly, commercial antigens of beef and mutton were used in the skin-prick test to detect red meat sensitivity (Allergopharma, Reinbek, Germany). The fresh red meat was used in the prick-to-prick test to those who responded negatively to the commercial allergen solution or responded marginally.,19 Histamine (10 $\mathrm{mg} / \mathrm{mL}$ ) was used as positive control, and normal saline was used as negative control. If induration was $3 \mathrm{~mm}$ or higher in the prick test, the patient was considered sensitive. Raw beef and mutton were used as fresh meat (Fig. 1). In the presence of cooked red meat, prick-toprick test was applied with it as well. Female and male beef/mutton were used in the prickto-prick test for some patients who had given conflicting information about food allergy in the clinical history.

\section{Serum-specific IgE}

Beef specific-IgE (f27) and mutton specific- $\operatorname{IgE}$ (f88) were analyzed with Chemiluminescence Immunoassay (CLIA) method employing the IMMULITE® 2000 XPi (Siemens Healthcare Diagnostics Inc., Deerfield, IL, USA) using $3 \mathrm{~g}$ Allergy ${ }^{\circledR}$ kits. The detection range for specific $\operatorname{IgE}$ (sIgE) was $\geq 0.10-100 \mathrm{kU} / \mathrm{L}$. The cutoff used for a positive test in these assays was $0.10 \mathrm{kU} / \mathrm{L}$. 

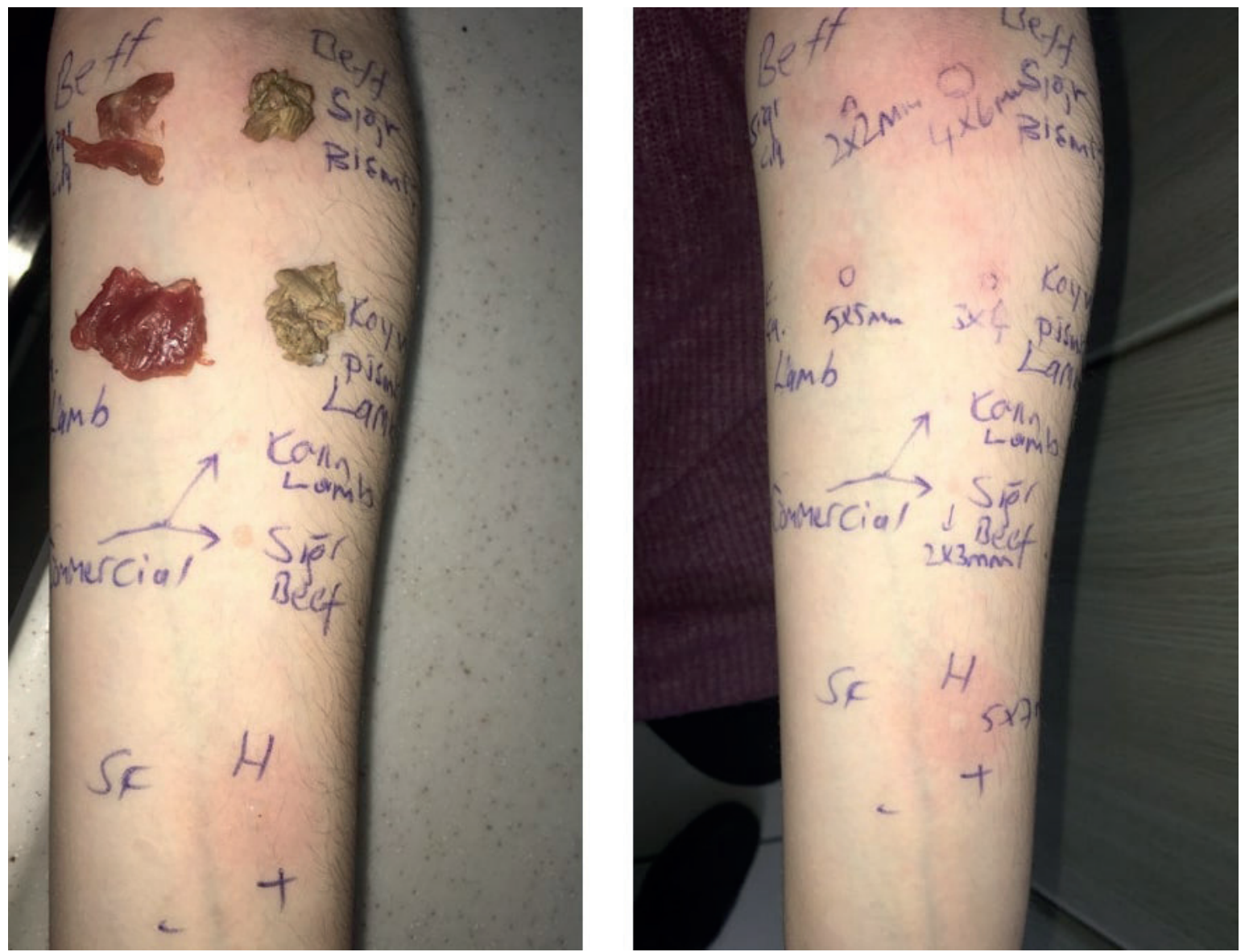

Fig. 1. The prick-to-prick test with raw and cooked red meat to detect red meat sensitivity in Patient 1.

\section{Other laboratory records}

In addition, the total IgE, eosinophil counts, hemoglobin, vitamin $\mathrm{D}\left(25 \mathrm{OH}-\mathrm{D}_{3}\right)$ level and blood groups of the patients were recorded.

\section{Oral Food Challenges}

The gold standard for food allergy diagnosis is double-blind placebo-controlled oral food challenge (DBPCOFC) test. ${ }^{1}$ Our red meat allergy patients usually come from outside the city. For this reason, all our patients had preferred the open oral food challenge test (OFC). Beef and mutton are consumed as red meat in Turkey. Although our aim was to perform a challenging test on both meats, we left it to the consumption habit and request of the patient as to which red meat to use in the test. The OFC was prepared in line with current challenge protocols. ${ }^{12,16}$
The titrated dose was given separately for beef and mutton as 1, 2, 7, 15, 25, $50 \mathrm{~g}$ with $20 \mathrm{~min}$ intervals. It was completed as not to exceed 100 g. The patients were monitored for at least 6 hours after the OFC.

\section{Statistical Analysis}

Data were analyzed using IBM SPSS Statistics (22nd version, IBM Corp., NY, USA). Descriptive statistics were expressed as mean \pm standard error or median, depending on the distribution of variables. Categorical variables were expressed as numbers and percentages. The normality test of numerical variables was verified with the Shapiro-Wilk test. ChiSquare and Fisher's Exact Tests were used to compare categorical variables. In comparison of quantitative data, independent sample $t$-test was used for those with normal distributions, 
while nonparametric tests such as Mann Whitney-U and Kruskal-Wallis test were used in non-normal distributions of the data.

\section{Results}

Sixty-three people were admitted to the hospital with suspicion of red meat allergy between the dates mentioned above. In this study, records of 43 patients who were detected to be sensitive to red meat in the prick-to-prick test were presented. The demographic, clinical and laboratory data of the patients are presented in Table I. The median age of the 43 patients was $12(2-37)$, and $51 \%$ were male. A total of $86 \%$ of the patients were between the ages of 2 and 17 years. A total of $86 \%$ of child patients were from the Ordu-Giresun in Turkey.

The data of the patients are summarized in Table II. All patients reported skin manifestations, and half of them reported respiratory symptoms upon exposure to red meat. In clinical history, anaphylaxis was described in $67 \%$ of patients. The most common accompanying allergic disease was respiratory allergic diseases such as asthma and allergic rhinitis. There was no allergic disease in $1 / 3$ patients. Cow's milk allergy (CMA) was present in a two-year-old patient (patient number 29). CMA was diagnosed at 6 months of age based on convincing clinical history and skinprick test results. The remaining 42 patients were consuming dairy products without any problems. Two of these 42 patients reported having CMA in infancy (patient number 21 and 22).

The mean age of the groups with and without anaphylaxis history were similar $(p=0.136)$. There were no significant relationship between the presence of anaphylaxis history and sex, family history and the delay time in diagnosis ( $p=0.586, \quad p=0.916, \quad p=0.175$, respectively). Although all the patients with parental consanguinity had anaphylaxis history, $69.6 \%$ of those who did not have parental consanguinity had anaphylactic history; however, this was not at a statistically significant level $(\mathrm{p}=0.146)$. No significant relationships were detected between the presence of anaphylaxis history and the presence of additional allergic disease, tick bite history, and mutton or beef sensitivity in the skin test ( $p=0.739, p=0.689, p=0.537$, respectively). No significant relationships were detected between the presence of anaphylaxis history and sIgE, total IgE, eosinophil count, and vitamin $\mathrm{D}$ level $(\mathrm{p}=1.00, \mathrm{p}=0.48, \mathrm{p}=0.34, \mathrm{p}=0.66$, respectively).

The presence of a tick bite history did not affect history of early-anaphylaxis or delayedanaphylaxis $(p=1.00)$. No relationships were detected between early or delayed-anaphylaxis history and age, and sensitivity of mutton or beef in prick-to-prick test $(p=1.00, p=0.580$, respectively). Although the number of children with early-anaphylaxis history between 0-6 years of age was 1.5 times higher than those who had early-anaphylaxis history at the age of 6-18 years, the difference was not significant $(\mathrm{p}=0.06)$.

There was no significant relationship between the presence of additional allergic diseases and the sensitivity of mutton or beef, and it was not associated with vitamin D levels ( $p=0.397$, $\mathrm{p}=0.184$, respectively). There was no significant relationship between mutton sensitivity in the skin test and mutton $\mathrm{sIgE}(\mathrm{p}=0.442)$. Similarly, no relationship was determined between beef sensitivity in skin test and beef $\operatorname{sgE}(\mathrm{p}=0.157)$.

The results of the patients that underwent the OFC are given in Table III. OFC was positive in 6 of the 9 patients. Three of 6 patients had developed anaphylaxis. Patient 36 had described moderate anaphylaxis after red meat consumption in his clinical history. He also had a history of vespula venom anaphylaxis. His sister had red meat allergy. He was applied the prick-to-prick test with beef. Since severe early-anaphylaxis developed after the skin test, the OFC test was not performed. The patient was advised to avoid all red meats. Adrenaline autoinjector was prescribed. Patient 1 , who had only urticaria symptoms after eating red meat, did not develop a reaction after OFC with beef. 
Table I. Characteristics of children and adults reporting reactions to red meat and sensitizied to red meat.

\begin{tabular}{|c|c|c|c|}
\hline Characteristics & $\begin{array}{c}\text { Total } \\
n=43(\%)\end{array}$ & $\begin{array}{c}\text { Children } \\
\mathrm{n}=37(86 \%)\end{array}$ & $\begin{array}{c}\text { Adult } \\
\mathrm{n}=6(4 \%)\end{array}$ \\
\hline Age, y, median (min-max) & $12(2-37)$ & $10(2-17)$ & $28(22-37)$ \\
\hline Sex, male (\%) & $22(51.2 \%)$ & $20(54.1 \%)$ & $2(33.3 \%)$ \\
\hline \multicolumn{4}{|l|}{ Clinical history, symptom/sign } \\
\hline Urticaria or urticaria and angioedema & $43(100)$ & $37(100)$ & $6(100)$ \\
\hline Respiratory (nasal congestion, cough, dyspnea) & $24(55)$ & $19(51)$ & $5(83)$ \\
\hline Heart (tachycardia, bradycardia, collapse) & $4(9)$ & $3(8)$ & $1(16)$ \\
\hline GIS (vomiting, diarrhea) & $8(18)$ & $6(16)$ & $2(33)$ \\
\hline Anaphylaxis & $29(67)$ & $24(64)$ & $5(83)$ \\
\hline Early (0-2 hours) & $23(53)$ & $19(51)$ & $4(66)$ \\
\hline Delayed (2-6 hours) & $6(13)$ & $5(13)$ & $1(16)$ \\
\hline Age of onset, median (min-max), year & $5(1-20)$ & $4(1-14)$ & $7.5(5-20)$ \\
\hline Delay time in diagnosis, median (min-max), year & $4(0-28)$ & $4(0-15)$ & $23(4-28)$ \\
\hline \multicolumn{4}{|l|}{ Timing of the symptoms in the clinical history } \\
\hline $0-2$ hours & $34(79)$ & $29(78)$ & $5(83)$ \\
\hline$>2$ hours & $9(21)$ & $8(22)$ & $1(16)$ \\
\hline Family history & $21(48)$ & $15(40)$ & $6(100)$ \\
\hline Parental consanguinity & $8(18)$ & $7(18)$ & $1(16)$ \\
\hline \multicolumn{4}{|l|}{ City of residence } \\
\hline Ordu-Giresun & $38(88)$ & $32(86)$ & $6(100)$ \\
\hline Other cities & $5(11)$ & $5(13)$ & $0(0)$ \\
\hline \multicolumn{4}{|l|}{ Comorbid allergic disease } \\
\hline Asthma or allergic rhinitis & $23(53)$ & $19(51)$ & $4(66)$ \\
\hline Atopic dermatitis or chronic spontan urticaria & $9(20)$ & $8(21)$ & $1(16)$ \\
\hline Vespula or bee venom allergy & $3(7)$ & $1(2)$ & $2(33)$ \\
\hline Cow's milk protein allergy & $1(2)$ & $1(2)$ & $0(0)$ \\
\hline No & $16(37)$ & $14(37)$ & $2(33)$ \\
\hline History of tick exposure & $18(41)$ & $14(37)$ & $4(66)$ \\
\hline \multicolumn{4}{|l|}{ Positivity in skin-prick test } \\
\hline Commercial antigen solution & $3(7)$ & $3(8)$ & $0(0)$ \\
\hline Prick-to-prick & $43(100)$ & $37(100)$ & $6(100)$ \\
\hline Beef only & $5(11)$ & $4(11)$ & $1(16)$ \\
\hline Mutton only & $6(13)$ & $5(14)$ & $1(16)$ \\
\hline Beef and mutton & $32(76)$ & $28(75)$ & $4(68)$ \\
\hline Total IgE (IU/mL), median (min-max) & $355(20-3550)$ & $327(20-3550)$ & $431(33-1100)$ \\
\hline \multicolumn{4}{|l|}{ Specific immunoglobulin E (kIU/L), } \\
\hline Mutton, median (min-max), n=18 & $0(0-7.25)(n=18)$ & $0(0-7.25)(\mathrm{n}=17)$ & $0(\mathrm{n}=1)$ \\
\hline Beef, median (min-max), n=18 & $0.21(0-31.2)(\mathrm{n}=18)$ & $0.23(0-31.2)(\mathrm{n}=17)$ & $0.2(\mathrm{n}=1)$ \\
\hline Eosinophil count $\left(\right.$ cell $\left./ \mathrm{mm}^{3}\right)$, median (min-max) & $210(40-990)$ & $210(40-990)$ & $170(40-320)$ \\
\hline Hemoglobin $(\mathrm{gr} / \mathrm{L})$, mean \pm standart error & $12.9 \pm 0.20$ & $12.8 \pm 0.17$ & $13.2 \pm 0.99$ \\
\hline $25 \mathrm{OH}-\mathrm{D}_{3}(\mathrm{mcg} / \mathrm{L})$, mean \pm standart error & $13.3 \pm 1.1(n=32)$ & $13.1 \pm 1.2(n=27)$ & $14.5 \pm 3.4(n=5)$ \\
\hline
\end{tabular}




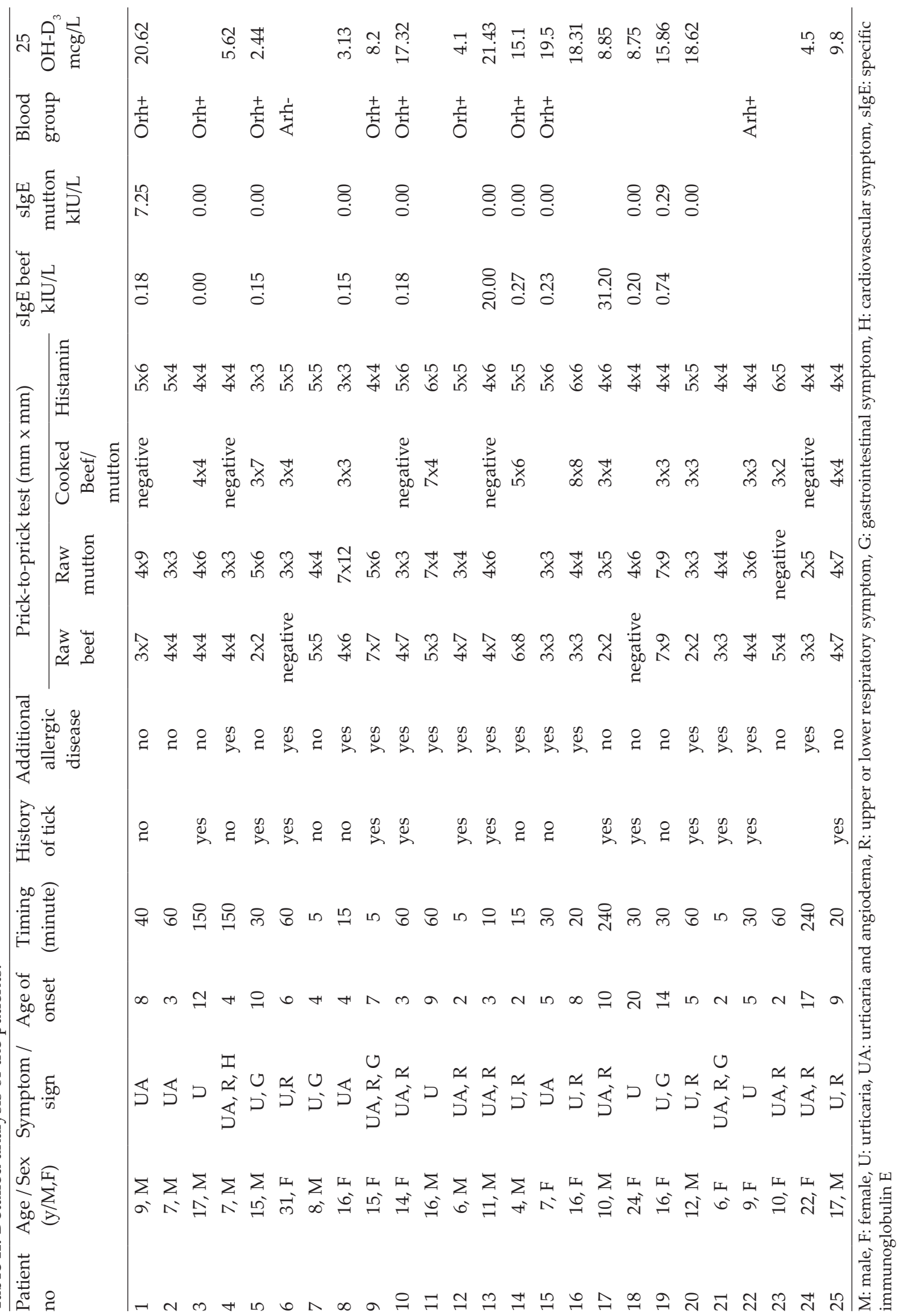




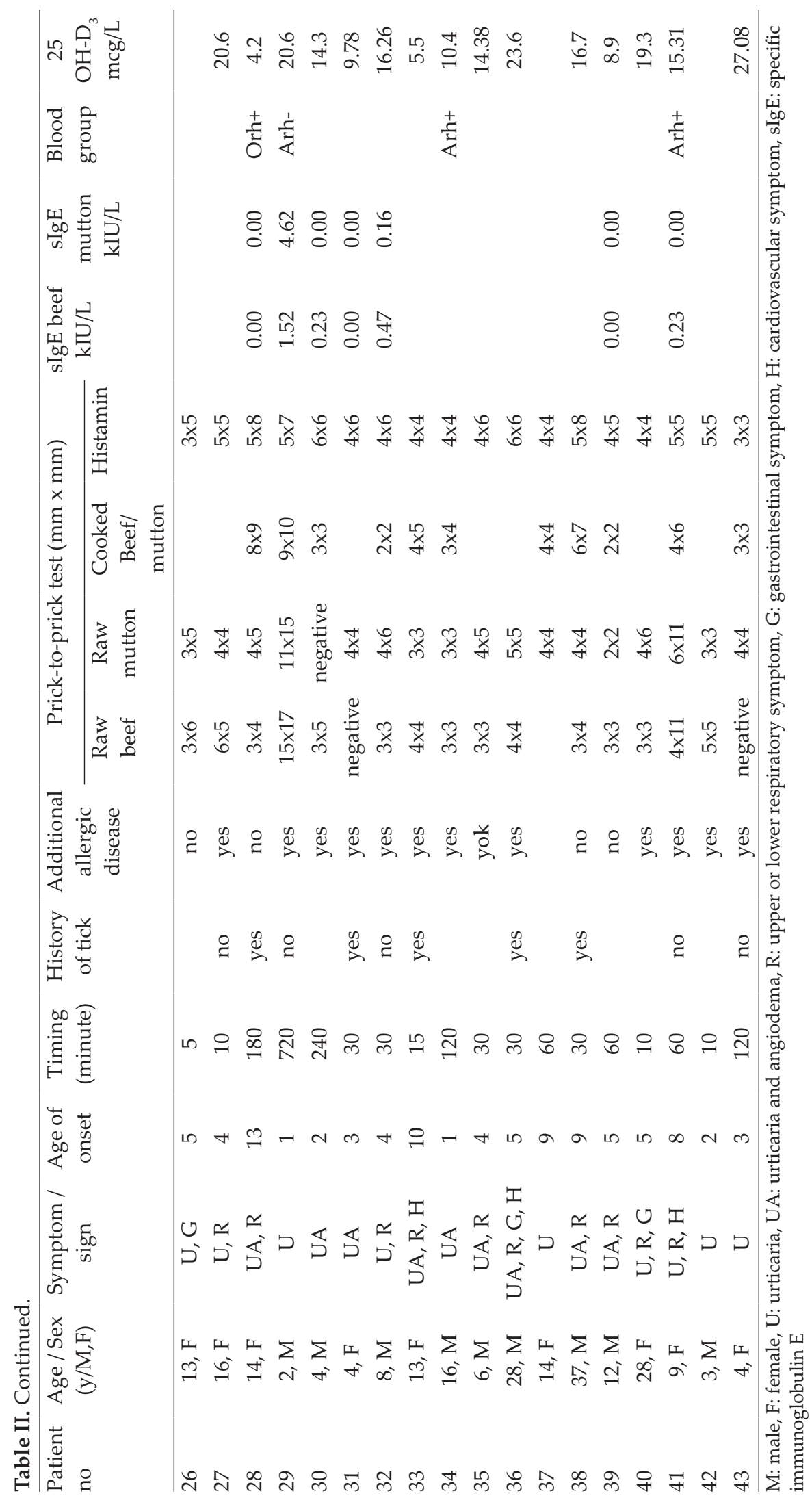




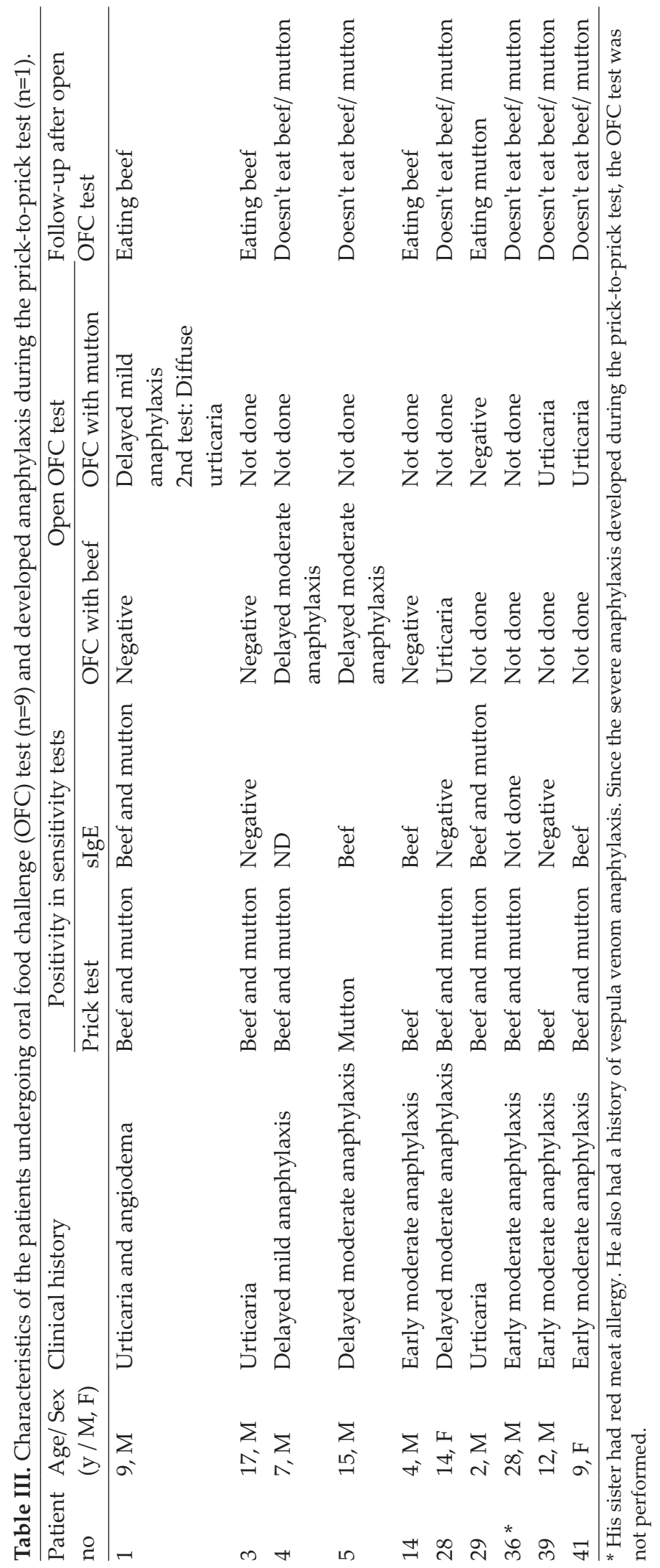


Then OFC was conducted with mutton at a different time. Six hours later, the patient had widespread urticaria, nasal congestion, runny nose, and sneezing. Anaphylaxis training was provided to the patient, and adrenaline autoinjector was prescribed. The patient said that he wanted to eat mutton and applied the second OFC. Then, the second OFC was done at a different time. Widespread urticaria developed four hours after OFC. There were no other system involvements. However, he was told that the risk of anaphylaxis continued, and to avoid mutton.

All the patients who underwent the OFC test were questioned by phone twice within one year. It was found that the 4 patients who had negative results in the OFC test could eat the recommended red meat. Also, some patients who described mild anaphylaxis in their clinical history (i.e. patients $15,35,40$ ) reported that they could eat red meat with antihistamines without any problems. Some patients (i.e. patient 3 and 19) reported that they had symptoms when they consumed large amounts of red meat. All patients who underwent the OFC test or reported anaphylaxis history were given anaphylaxis training, and adrenaline autoinjectors were prescribed.

\section{Discussion}

In this retrospective study, which included the highest number of red meat allergy patients in Turkey, the clinical characteristics of our patients were summarized. Most of our patients had an anaphylaxis history. The clinical and laboratory findings were very heterogeneous. These findings are not as helpful as the OFC test for diagnosis. The positivity and anaphylaxis rates in OFC tests were higher than other food allergies. ${ }^{12,13,15}$ Also, the mutton and beef sensitivity in the prick-to-prick tests were evaluated separately in this study for the first time. In some red meat allergy patients, mutton or beef may be recommended as an alternative to red meat after OFC test.
Three types of diseases are reported for red meat allergy, which are primarily beef allergy, pork-cat syndrome and alpha-gal syndrome. ${ }^{8}$ Although red meat allergy is rarely reported among other food allergies in the world, the case reports with alpha-gal syndrome have increased in recent years. ${ }^{20-22}$ Recently identified alphagal syndrome is the appearance of anaphylaxis findings and symptoms 4-6 hours after red meat consumption with galactose-alpha-1.3galactose (alpha-gal) sIgE, caused by the bite of some tick species, ${ }^{19,23}$ which was included in the literature as the cause of delayed-anaphylaxis. ${ }^{19}$ Alpha-gal sIgE was not measured in our case series. Our data were retrospectively collected. Alpha-gal syndrome is usually reported in adults, ${ }^{8}$ and is reported very rarely in Turkey. ${ }^{21,22}$ It should also be considered that alpha-gal sIgE might be elevated without red meat allergy, due to parasitic helminth infection, tick allergy, or a monoclonal antibody (i.e. cetuximab) allergy. ${ }^{23}$ A total of $13 \%$ of our case series had reported delayed-anaphylaxis. We cannot be sure about the tick bite history especially in children. Awareness of tick bite has increased in our region due to Crimean Congo hemorrhagic fever disease. We questioned the tick bite history in detail from their parents. We did not find a relationship between tick bite history and presence of delayed-anaphylaxis history. No differences were reported even in individuals with alpha-gal syndrome in terms of alphagal sIgE among those who reported early and delayed-anaphylaxis. ${ }^{20}$ Since there is no pork consumption in Turkey, pork-cat syndrome has not been reported so far.

Our patients with red meat allergy are primary beef allergies according to prick-to-prick tests. Likewise, another study in our region reported that children with red meat allergies had primarily beef allergies. ${ }^{16}$ They conducted this study in the Eastern Black Sea region in Turkey, which included 4932 school children, the prevalence of primary beef allergy was reported to be $2.6 \%$ based on a questionnaire, and $0.3 \%$ based on the OFC test. ${ }^{16}$ Most of our patients were from the Eastern Black Sea region 
(Ordu-Giresun). In this prevalence study, reactions in the first 2 hours were recorded, whereas reactions between 2-6 hours were not recorded. Symptoms and signs may have developed after 2-6 hours in some of our patients undergoing OFC. For this reason, the expected red meat allergy prevalence in our region might be higher. In another prevalence study on challenge-proven food allergy in school children in the Eastern Black Sea Region, the most commonly detected food allergy was beef allergy, and then, milk, cocoa and egg allergies. ${ }^{12}$ In another multi-centric prevalence study on food allergy in children in Turkey, it was reported that beef allergy was the second most frequent food allergy. ${ }^{13}$ This information is different from studies conducted in western populations. Red meat allergy prevalence may vary according to geographical regions. ${ }^{7,20}$ Red meat allergy awareness should be increased among pediatricians, at least in Turkey . In our patients, the age of onset of red meat allergy was approximately 4 years of age. This result was similar to the literature data. ${ }^{14,16,24}$ The delay in diagnosis was 4 years. As reported previously, these patients were diagnosed late. ${ }^{17}$

Skin manifestations were reported in 66-93\% of patients with red meat allergy. ${ }^{16,20}$ In our case series, all patients had skin symptoms. Since our hospital is a tertiary hospital, not all patients might have been referred to us. We did not have any patients with only gastrointestinal tract (GIS) symptoms. Presentation with only GIS symptoms is reported rarely in the literature. ${ }^{20}$ Food intolerance or poisoning may be considered in those who report complaints 4-6 hours after food intake. However, if the symptom repeats in the same person, or if only one of the few people who have taken the same food has symptoms, a food allergy should be considered. Food allergy is usually not considered in chronic spontaneous urticaria (CSU). ${ }^{25}$ However, it was reported that we should consider red meat allergy disease in differential diagnosis in recurrent urticaria or in CSU. ${ }^{16,20}$ Some of our patients had recurrent urticaria. Clinical variability may occur in patients with red meat allergy. ${ }^{23,26}$ In these patients, clinical variability may not be due to potential cofactors (i.e. nonsteroid intake, alcohol, exercise) for food allergies. ${ }^{23,26}$ In the same person, exposures to red meat at different times may cause acute urticaria or anaphylaxis, or may not cause any symptoms. ${ }^{8,23,26}$ Clinical variability may depend on the amount of meat consumed, the allergen contained in the meat, and industrial processing. ${ }^{8,26} \mathrm{But}$, the exact cause of this is not yet known. For this reason, there is no single diagnostic algorithm for red meat allergy. ${ }^{8}$ Clinical history should be questioned carefully in these patients. Although it was not previously reported in the literature, we applied the prick-to-prick test with both female and male red meat to those who had contradictory clinical histories. Different sensitivity results in prick tests were detected in some patients. For this reason, it may be considered that one of the reasons for the variability in the history and laboratory findings might be due to the male/ female status of the red meat.

The most important result of this case series is that both positivity and anaphylaxis rates in the OFC test were higher than in other food allergies. ${ }^{12,13}$ This finding was also detected in beef allergic children in DBPCOFC test and in alpha-gal syndrome patients in open OFC test. ${ }^{16,27}$ Also, it was reported that red meat allergies should be considered in idiopathic anaphylaxis patients. ${ }^{17,18}$ We did not find a relationship between anaphylaxis history and demographic information, clinical history and skin tests of the patients. There is no study on predicting anaphylaxis in these patients. Some of our patients said that they could eat red meat by using antihistamines even if they had described mild anaphylaxis history. Similar notification is available in the literature. ${ }^{28}$ However, precautions should be taken for anaphylaxis, due to clinical variability.

Skin test and $\operatorname{sgE}$ are recommended as sensitivity tests in patients with suspected red meat allergy. ${ }^{8}$ Some researchers report that sIgE is more sensitive than prick tests. ${ }^{14,16}$ However, in our challenge-proven patients, mutton and beef 
sIgE were negative in some. In our series, very few patients had sensitivity with commercial allergen in the skin test. In these patients, skin testing is recommended with fresh meat rather than commercial allergens. ${ }^{19}$ Skin test and sIgE can be negative in some challenge-proven red meat allergy patients. ${ }^{16}$ Furthermore, beef sensitivity tests may be negative in some patients who have had anaphylaxis with beef. ${ }^{12}$ In our series, mutton $\operatorname{sIgE}$ was negative in 2 patients who had mutton allergy in the OFC test. It was reported that mutton SIgE is less sensitive than beef $\operatorname{sIgE} .^{29}$ There is very heterogeneity in laboratory results in red meat allergy patients. ${ }^{16,20,29}$ In the present study, no correlation was detected between the skin test and sIgE both for beef and mutton. Although red meat is not essential for a diet, it is more important for children and adolescents than adults. $^{7}$ Therefore, we wanted to recommend alternative red meat as mutton or beef for red meat allergic children. Furthermore, it is difficult for children to avoid allergens to which they are allergic. ${ }^{3}$ We also performed the skin test and OFC test for beef and mutton separately. Some of our patients were able to eat alternative red meat without any problems after the OFC test. There is no study suggesting another red meat as an alternative in these patients. ${ }^{8}$

Most of our patients had other allergic diseases, the most common of which were respiratory allergies. It was reported that allergic diseases may be common in primary beef allergy patients. ${ }^{8}$ In our case series, a patient with vespula venom allergy developed severe anaphylaxis during the prick-to-prick test. For this reason, if a patient with vespula venom allergy is performed a skin test or OFC test for red meat allergy research, extreme caution should be exercised for anaphylaxis. We could not measure the tryptase level in the patient to investigate the mast cell disorder. Recently, a combination of red meat allergy and venom allergy has been reported. ${ }^{30}$ However, there is no clear explanation yet about the reason for this combination. It was reported previously that milk allergy was common in patients with beef allergy. ${ }^{31}$ However, cow's milk allergy in our pediatric patients was not more common than in the general population. This finding is consistent with other reports in Turkey. ${ }^{14,16}$ The rate of parental consanguinity in our patients was similar to the overall rate reported in Turkey. Half of our patients had a family history of allergies. There was a similar finding in another study in Turkey. ${ }^{16}$ This finding suggests that polygenic and environmental factors may also be effective in red meat allergy, as in other allergic diseases.

Vitamin D levels of red meat allergy patients have never been reported. Vitamin D level and effect in allergic diseases continues to be investigated. ${ }^{32,33}$ The mean vitamin $\mathrm{D}$ level of our pediatric patients was $13.1 \mathrm{mcg} / \mathrm{L}$. Since this study was retrospective, we did not compare it with concurrent controls. However, the mean vitamin D level previously reported in healthy children of similar age and in the same geographic region was $16.9 \mathrm{mcg} / \mathrm{L} .{ }^{32}$ Low vitamin D levels compared to healthy children may be an issue that needs to be investigated prospectively. Our pediatric patients were not anemic despite avoiding eating red meat for a long time. They were not taking any iron replacement. This issue was not addressed in previous reports. We could not explain this unexpected finding. Unfortunately, we did not question the daily diet of the patients. It can be thought that iron sources other than red meat in the diet may be sufficient to prevent anemia when taken enough. Patients with red meat allergy due to alpha-gal syndrome are reported to have a B-negative blood group. . $^{20,34}$ In our series, 14 patients tested for blood types had B-negative blood type. There are no reports of blood group of individuals with primary beef allergy in the literature. The antigens responsible for primary beef allergy (bovine serum albumin, immunoglobulin, myosin light chain kinase, parvalbumin, enolase, aldolase) should be compared biochemically with the blood group antigens. ${ }^{8}$

It was reported that the basophile activation test may be an alternative to OFC test in 
patients with red meat allergy due to alpha-gal syndrome. ${ }^{35}$ No study has been performed on this issue in patients with primary beef allergies. The most important limitation of our report was that the DBPCOFC test was not conducted. DBPCOFC is time-consuming and difficult to apply in a clinical setting. However, open OFC test can be used reliably in daily allergy practice in food allergies. ${ }^{36}$ It was reported in the literature that the results of the open OFC test in red meat allergies were compatible with clinical and laboratory findings. ${ }^{14,27}$ The second important limitation was that alpha-gal sIgE was not measured. Another limitation was that some allergic diseases reported to be associated in patients with red meat allergy (such as cow's milk allergy, cat dander, pork meat) were not investigated.

As a result, clinical history and sensitivity tests are important in patients with red meat allergy, as in all food allergies. However, they are not enough in red meat allergy. There are clinical and laboratory variability in these patients. This complicates the diagnostic algorithm. OFC testing is useful not only to confirm the diagnosis but also to suggest an alternative to red meat. More severe findings may occur in the OFC test compared to the clinical history. The risk of anaphylaxis in these patients is higher than in patients with other food allergies. Anaphylaxis does not mean that it will not develop in subsequent reactions, even if it is only a symptom of urticaria. Red meat allergy patients can respond differently to each repeated OFC test, therefore, should be followed even after negative OFC test. Anaphylaxis training and adrenaline autoinjector should be given to those who develop anaphylaxis in the OFC test and to red meat sensitive patients who report anaphylaxis in their clinical history.

\section{Acknowlegement}

We would like to thank the pediatric nurse Sat1 Aykut for help in the prick-to-prick test.

\section{Author contribution}

The authors confirm contribution to the paper as follows: study conception and design: study conception and design: ŞK, FÖ; data collection: ŞK, GH, ŞİKK; analysis and interpretation of results: ŞK, GH, FÖ, RS; draft manuscript preparation: ŞK, RS, FÖ. All authors reviewed the results and approved the final version of the manuscript.

\section{Ethical approval}

The study was approved by the Ondokuz Mayis University Ethics Committee of our institution (KAEK 2017/55).

\section{Source of funding}

There is no sources of financial assistance.

\section{Conflict of interest}

The authors declare no conflict of interest.

\section{REFERENCES}

1. Abrams EM, Sicherer SH. Diagnosis and management of food allergy. CMAJ 2016; 188: 10871093.

2. Sicherer SH, Sampson HA. Food allergy: a review and update on epidemiology, pathogenesis, diagnosis, prevention, and management. J Allergy Clin Immunol 2018; 141: 41-58.

3. Mehta H, Groetch M, Wang J. Growth and nutritional concerns in children with food allergy. Curr Opin Allergy Clin Immunol 2013; 13: 275-279.

4. Keet CA, Savage JH, Seopaul S, Peng RD, Wood RA, Matsui EC. Temporal trends and racial/ethnic disparity in self-reported pediatric food allergy in the United States. Ann Allergy Asthma Immunol 2014; 112: 222-229.e3.

5. Lieberman $P$, Nicklas RA, Randolph $C$, et al. Anaphylaxis-a practice parameter update 2015. Ann Allergy Asthma Immunol 2015; 115: 341-384.

6. Steinke M, Fiocchi A, Kirchlechner V, et al; REDALL study consortium. Percevied food allergy in children in 10 European nations. A randomised telephone survey. Int Arch Allergy Immunol 2007; 143: 290295. 
7. Restani P, Ballabio C, Tripodi S, Fiocchi A. Meat allergy. Curr Opin Allergy Clin Immunol 2009; 9: 265-269.

8. Wilson JM, Platts-Mills TAE. Red meat allergy in children and adults. Curr Opin Allergy Clin Immunol 2019; 19: 229-235.

9. Doğruel D, Bingöl G, Altıntaş DU, Yılmaz M, Güneşer Kendirli S. Clinical features of food allergy during the 1st year of life: the ADAPAR birth cohort study. Int Arch Allergy Immunol 2016; 169: 171-180.

10. Barlık F, Güner ŞN, Barlık M, Söğüt A, Sancak R. Prevalence of food allergy in nursery and kindergarten children in Samsun. Turk Arch Pediatr 2013; 48: 288-293.

11. Kaya A, Erkoçoğlu M, Civelek E, Çakır B, Kocabaş $\mathrm{CN}$. Prevalence of confirmed IgE-mediated food allergy among adolescents in Turkey. Pediatr Allergy Immunol 2013; 24: 456-462.

12. Orhan F, Karakas T, Cakir M, Aksoy A, Baki A, Gedik Y. Prevalence of immunoglobulin E-mediated food allergy in 6-9-year-old urban schoolchildren in the eastern Black Sea region of Turkey. Clin Exp Allergy 2009; 39: 1027-1035.

13. Mustafayev R, Civelek E, Orhan F, Yüksel H, Boz AB, Sekerel BE. Similar prevalence, different spectrum: IgE-mediated food allergy among Turkish adolescents. Allergol Immunopathol (Madr) 2013; 41: 387-396.

14. Orhan F, Sekerel BE. Beef allergy: a review of 12 cases. Allergy 2003; 58: 127-131.

15. Orhan F, Canitez Y, Bakirtas A, et al. Anaphylaxis in Turkish children: a multi-centre, retrospective, case study. Clin Exp Allergy 2011; 41: 1767-1776.

16. Topçu ZIKK, Kaklıkkaya N, Baki A, Orhan F. Characteristics of beef allergy in schoolchildren in Turkey. Allergy Asthma Proc 2018; 39: 59-65.

17. Flaherty MG, Kaplan SJ, Jerath MR. Diagnosis of life-threatening alpha-gal food allergy appears to be patient driven. J Prim Care Community Health 2017; 8: 345-348.

18. Pattanaik D, Lieberman P, Lieberman J, Pongdee $\mathrm{T}$, Keene AT. The changing face of anaphylaxis in adults and adolescents. Ann Allergy Asthma Immunol 2018; 121: 594-597.

19. Commins SP, Satinover SM, Hosen J, et al. Delayed anaphylaxis, angioedema, or urticaria after consumption of red meat in patients with $\operatorname{IgE}$ antibodies specific for galactose-alpha-1,3-galactose. J Allergy Clin Immunol 2009; 123: 426-433.
20. Wilson JM, Schuyler AJ, Workman L, et al. Investigation into the $\alpha$-Gal syndrome: characteristics of 261 children and adults reporting red meat allergy. J Allergy Clin Immunol Pract 2019; 7: 2348-2358.e4.

21. Unal D, Coskun R, Demir S, Gelincik A, Colakoglu B, Buyukozturk S. Successful beef desensitization in 2 adult patients with a delayed-type reaction to red meat. J Allergy Clin Immunol Pract 2017; 5: 502-503.

22. Keleş Ş, Gündüz M. Alpha gal specific IgE positivity due to tick bites and red meat allergy: the first case report in Turkey. Turk J Pediatr 2019; 61: 615-617.

23. Steinke JW, Platts-Mills TA, Commins SP. The alphagal story: lessons learned from connecting the dots. J Allergy Clin Immunol 2015; 135: 589-596.

24. Fiocchi A, Restani P, Riva E. Beef allergy in children. Nutrition 2000; 16: 454-457.

25. Zuberbier T, Bernstein JA. A comparison of the United States and International perspective on chronic urticaria guidelines. J Allergy Clin Immunol Pract 2018; 6: 1144-1151.

26. Wilson JM, Platts-Mills TAE. Meat allergy and allergens. Mol Immunol 2018; 100: 107-112.

27. Commins SP, James HR, Stevens W, et al. Delayed clinical and ex vivo response to mammalian meat in patients with IgE to galactose-alpha-1,3-galactose. J Allergy Clin Immunol 2014; 134: 108-115.

28. Heber G, Abraham S, Bauer A. Urticaria and angioedema after consumption of red meat. J Dtsch Dermatol Ges 2013; 11: 673-674.

29. Brestoff JR, Zaydman MA, Scott MG, Gronowski AM. Diagnosis of red meat allergy with antigenspecific IgE tests in serum. J Allergy Clin Immunol 2017; 140: 608-610.e5.

30. Fischer J, Huynh HN, Hebsaker J, Forchhammer S, Yazdi AS. Prevalence and impact of type I sensitization to alpha-gal in patients consulting an allergy unit. Int Arch Allergy Immunol 2020; 181: 119-127.

31. Martelli A, De Chiara A, Corvo M, Restani P, Fiocchi A. Beef allergy in children with cow's milk allergy; cow's milk allergy in children with beef allergy. Ann Allergy Asthma Immunol 2002; 89(6 Suppl 1): 38-43.

32. Kutluğ S, Kılıç M, Bilgici B, Paksu Ş, Yıldıran A, Sancak R. An evaluation of vitamin D levels in children with seasonal allergic rhinitis during pollen season. Pediatr Allergy Immunol 2017; 28: 446-451.

33. Yepes-Nuñez JJ, Brożek JL, Fiocchi A, et al. Vitamin D supplementation in primary allergy prevention: systematic review of randomized and nonrandomized studies. Allerg 2018; 73: 37-49. 
34. Hamsten C, Tran TA, Starkhammar M, et al. Red meat allergy in Sweden: association with tick sensitization and B-negative blood groups. J Allergy Clin Immunol 2013; 132: 1431-1434.

35. Mehlich J, Fischer J, Hilger C, et al. The basophil activation test differentiates between patients with alpha-gal syndrome and asymptomatic alpha-gal sensitization. J Allergy Clin Immunol 2019; 143: 182189.
36. Lieberman JA, Cox AL, Vitale M, Sampson HA Outcomes of office-based, open food challenges in the management of food allergy. J Allergy Clin Immunol 2011; 128: 1120-1122. 